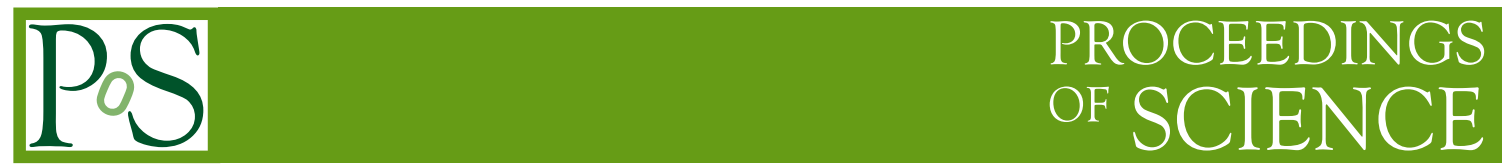

\title{
SiPM Photodetectors for Highest Time Resolution in PET
}

\author{
S. Gundacker*, E. Auffray, B. Frisch, T. Meyer, P. Jarron, P. Lecoq \\ European Organization for Nuclear Research (CERN), 1211 Geneva 23, Switzerland \\ E-mail: stefan.gundackerecern.ch
}

\begin{abstract}
Silicon photomultipliers (SiPM) have a wide range of applications in high energy and medical detector physics. Their excellent timing properties and compactness make them particularly interesting for time of flight positron emission tomography (TOF-PET). This study aims at determining the optimum detector conditions for highest time resolution in a TOF-PET system. The measurements are based on the time over threshold method in a coincidence setup using the ultra-fast amplifier-discriminator NINO and a fast oscilloscope. We compare commercial SiPMs of different SPAD types (Hamamatsu MPPC S10931-025P, S10931-050P and S10931-100P) and production batches. To explore the intrinsic timing capabilities of the SiPM, we coupled short, i.e. $2 \times 2 \times 5 \mathrm{~mm}^{3}$, Ca-co-doped LSO:Ce crystals to the different MPPCs and obtained best results for the $50 \mu \mathrm{m}$ type of $142 \pm 4 \mathrm{ps}$ FWHM CTR. This performance makes SiPMs ideal candidates for photodetectors in high resolution TOF-PET systems. The results will be discussed in terms of SiPM dark noise and photon detection efficiency (PDE).
\end{abstract}

International Workshop on New Photon-detectors,

June 13-15, 2012

LAL Orsay, France

* Speaker. 


\section{Introduction}

Silicon photomultipliers (SiPMs) or multipixel photon counters (MPPCs) are interesting where timing issues are of importance. They are promising candidates for time of flight positron emission tomography (TOF-PET) [1, 2]. With 10mm long crystals, such detectors can achieve coincidence time resolutions (CTR) of about 200ps FWHM [3]. They are thus comparable and even better than the best values achieved with PMTs [4]. The travel jitter within the crystal is not a neglectable factor $[5,6]$. One can ommit its influence on the overall time resolution by using crystals of length smaller or equal to $5 \mathrm{~mm}$. In such conditions and for LSO:Ce a CTR of about 140ps FWHM is achievable with SiPMs [7]. The research performed so far highlights the strong advantages of SiPMs in terms of timing. Potential drawbacks are the high dark count, high temperature dependence and the need for very good electronics to amplify and read out the small signals generated by one pixel. This paper discusses the limits of different SiPMs from the producer Hamamatsu in terms of timing. We used only LSO:Ce codoped Ca crystals with a length of $5 \mathrm{~mm}$, and electronics developed at CERN.

\section{Setup}

We chose crystals of $2 \times 2 \times 5 \mathrm{~mm}^{3}$ to minimise the influence of time travel jitter due to different light paths in the crystal, and to be only limited by the scintillation mechanisms. The crystals were wrapped in Teflon and coupled with optical grease (Rhodorsil 47V) to the photodetector. The different SiPMs used are shown in Tab.1. For the 50 $\mu$ SPAD size type, we compare 2 different batches produced at different times and one selected MPPC with lower DCR (dark count rate).

\begin{tabular}{ccccc}
\hline $\begin{array}{c}\text { Type: } \\
\text { S10931 }\end{array}$ & $\begin{array}{c}\text { SPAD } \\
\text { size } \\
\left(\mu \mathrm{m}^{2}\right)\end{array}$ & $\begin{array}{c}\text { Number } \\
\text { of } \\
\text { Cells }\end{array}$ & $\begin{array}{c}\text { Fill } \\
\text { Factor } \\
(\%)\end{array}$ & $\begin{array}{c}\text { break } \\
\text { down } \\
(\mathrm{V})\end{array}$ \\
\hline \hline -100P (received June 2010) & $100 \times 100$ & 900 & 78.5 & 69.3 \\
-050P (received June 2010) & $50 \times 50$ & $3^{\prime} 600$ & 61.5 & 70.5 \\
-050P (received May 2011) & $50 \times 50$ & $3^{\prime} 600$ & 61.5 & 70.9 \\
-050P (impr. DCR, Jan. 2012) & $50 \times 50$ & $3^{\prime} 600$ & 61.5 & 70.6 \\
-025P (received June 2010) & 25x25 & $14^{\prime} 400$ & 30.8 & 69.2 \\
\hline
\end{tabular}

Table 1: Properties of the different photodetectors, HAMAMATSU with $3 \times 3 \mathrm{~mm}^{2}$ active area

We characterize the timing properties with the standard coincidence measurement [8] as seen in Fig.1,a. The SiPM signals are fed into the CERN developed NINO chip [9]. NINO is an ultrafast discriminator amplifier employing the "time over threshold" technique. It produces a squared output pulse with the leading edge determining the time information (leading edge discrimination) and the width being a function of the pulse charge and thus delivering energy information, see Fig.1,b. NINO uses a differential readout of the SiPM signals [10] and hence can actively suppress common ground noise and pick-up.

The entire setup was housed in a thermally insulated dark box. The temperature was held stable at $20 \pm 0.1^{\circ} \mathrm{C}$. This should avoid temperature induced gain fluctuations during a single CTR measurement. 


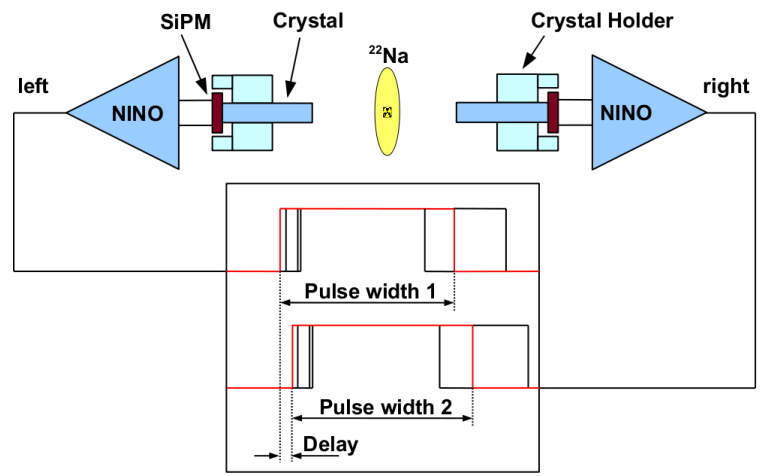

(a)

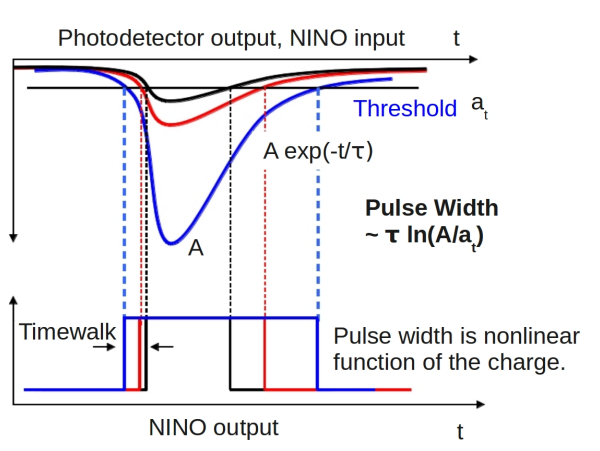

(b)

Figure 1: Test setup using NINO, (a) schematic of the coincidence time measurement and (b) generation of the NINO output signal from the SiPM input

The data acquisition was performed with a high sampling rate oscillocope, LeCroy DDA 735Zi (40GS/s). The dual pulse widths from the NINO outputs of each branch of the coincidence setup, plus their delay, were always recorded on disk [3]. In the offline data analysis the time delay histogram is plotted taking only delay events lying in the two photopeaks into account, Fig.2. This offline analysis could be done in real time by just selecting events with pulse widths belonging to energies in the photopeaks.
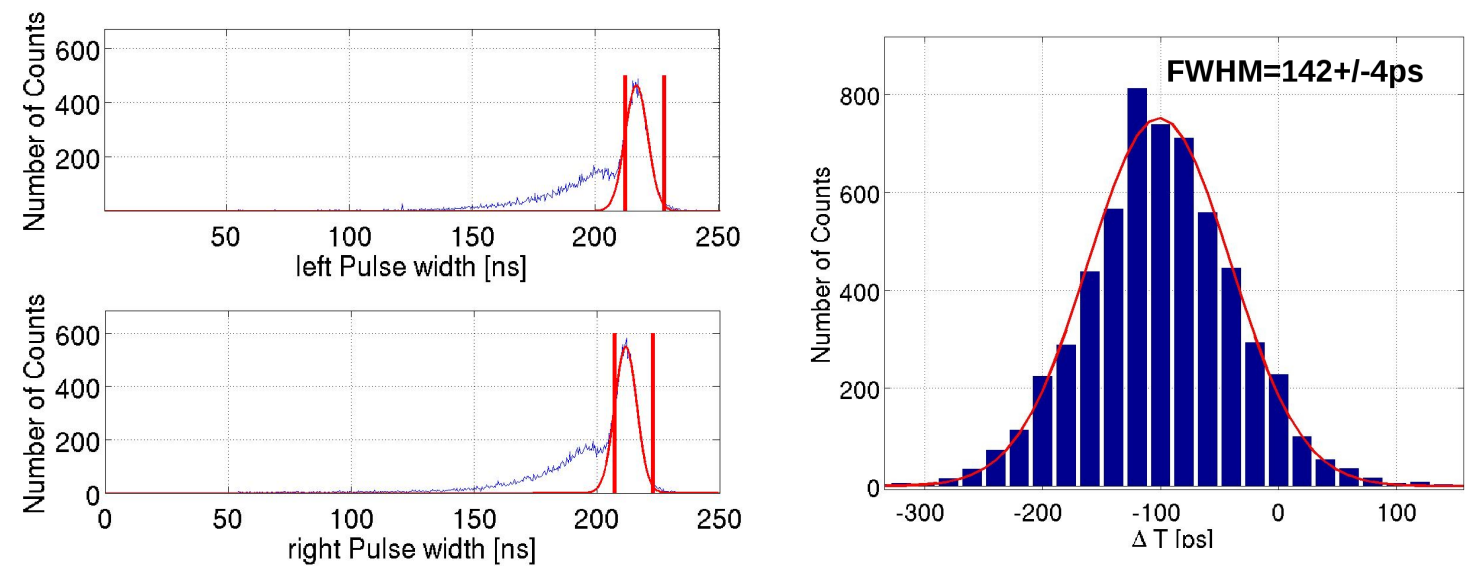

(a)

(b)

Figure 2: In the analysis we select (a) for both sides only delay events lying in both photopeaks of the NINO output pulses which is leading to (b) the time delay histogram from which we derive the CTR

It is also possible to define a reference detector with this setup. For that purpose we modify one branch of the setup. We glued a $2 \times 2 \times 5 \mathrm{~mm}^{2} \mathrm{LSO}$ :Ce:Ca crystal fully wrapped in Teflon on a Hamamatsu $50 \mu \mathrm{m}$ MPPC and always operated it at the same bias and threshold voltage. The reference was determined to have a time resolution of 107ps FWHM. By measuring a SiPM plus crystal under test versus this reference we obtain $C T R^{m}$. Aplying the formula $C T R=\sqrt{\left(C T R^{m}\right)^{2}-107^{2}} * \sqrt{2}$ one can calculate the CTR of the SiPM plus crystal under test. 


\section{Results for different SPAD sizes}

We made a series of systematic measurements changing the SiPM bias and NINO threshold voltage. We always used the same type of scintillator, i.e. LSO:Ce with $0.4 \%$ Ca codoping and $2 \times 2 \times 5 \mathrm{~mm}^{3}$ in dimensions, ensuring to investigate only the timing behaviour of the different SiPMs tested. Fig. 3 to Fig.5 show bias and threshold voltage scans made with different SiPMs of $25 \mu \mathrm{m}$, $50 \mu \mathrm{m}$ and $100 \mu \mathrm{m}$ SPAD size, all from the June 2010 batch. For every case we provided a staircase plot that shows the DCR over NINO threshold. The staircase plots give important information not only of the DCR value but also of the transition threshold between the $1^{\text {st }}$ and $2^{\text {nd }}$ SPADs firing.

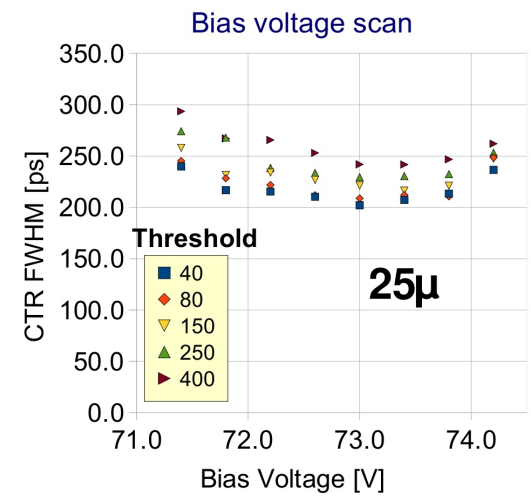

(a)

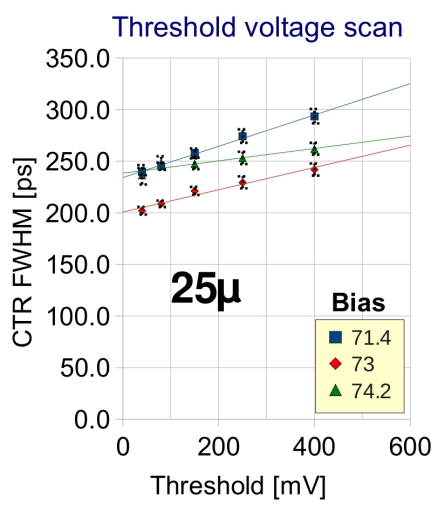

(b)

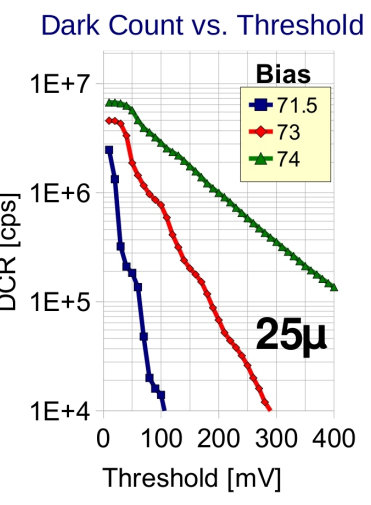

(c)

Figure 3: $25 \mu \mathrm{m}$ SPAD size: (a) bias voltage scan, (b) NINO threshold voltage scan, (c) DCR scan

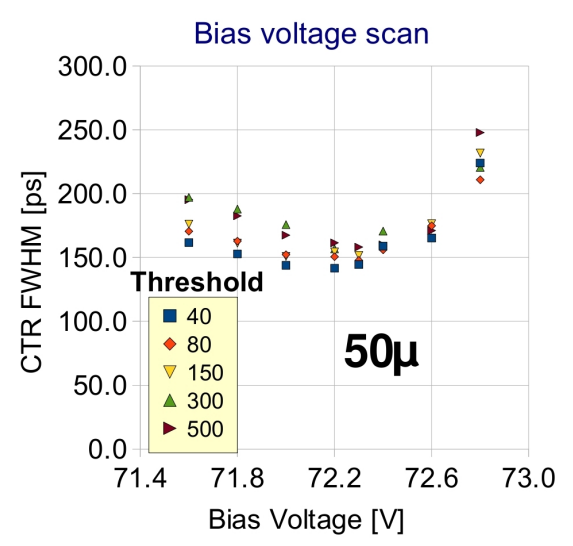

(a)

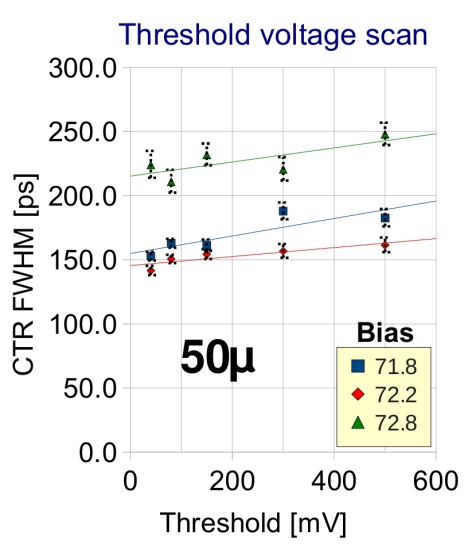

(b)

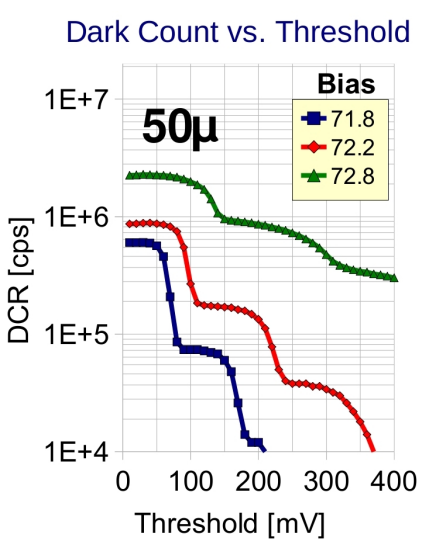

(c)

Figure 4: $50 \mu \mathrm{m}$ SPAD size: (a) bias voltage scan, (b) NINO threshold voltage scan, (c) DCR scan

All SiPMs have a minimum CTR seen in the bias voltage scans. This is due to the PDE being a product of geometrical fill factor, quantum efficiency and the probability of triggering an avalanche. With increasing bias voltage the avalanche triggering probability becomes higher, thus increasing the PDE. An increased PDE allows to detect more photons of the scintillation process and thus improves timing by improving statistics. Dark count rate is increasing with bias overvoltage as well. Above a certain bias overvoltage the PDE levels off and dark count begins to deteriorate the 
time resolution, giving rise to the observed minimum.

With decreasing NINO threshold, time resolution becomes better as a consequence of triggering at lower ranks of the scintillation's Poisson process. The fact that we do not see an increase in CTR at very low threshold levels implies a very low electronic noise level of NINO.

For the $100 \mu \mathrm{m}$ type and high bias voltage values we see an increase in CTR if going to lower threshold values, Fig.5,b. This can be explained by the very high DCR of this device adding baseline shifts and subsequently deteriorating the CTR.

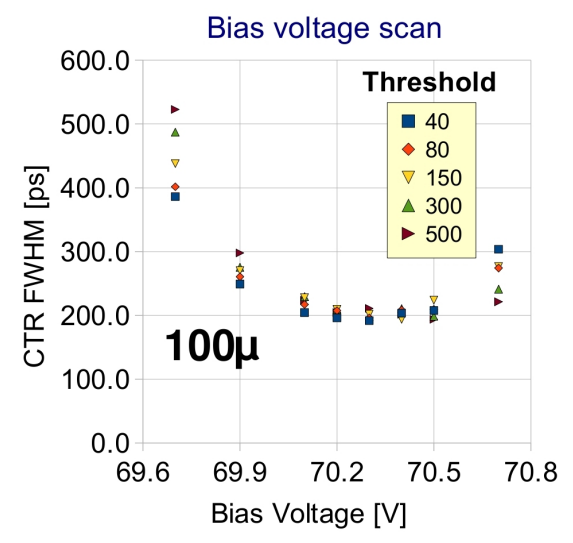

(a)

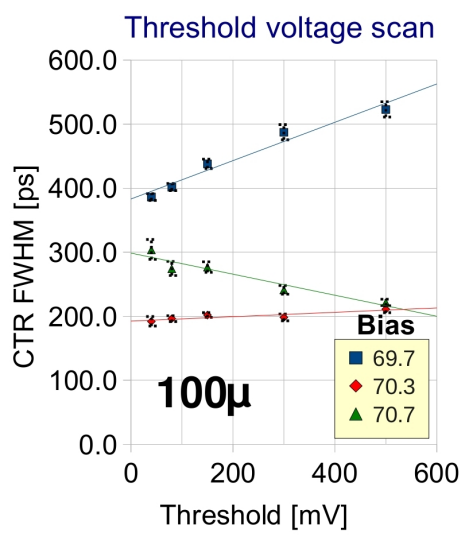

(b)

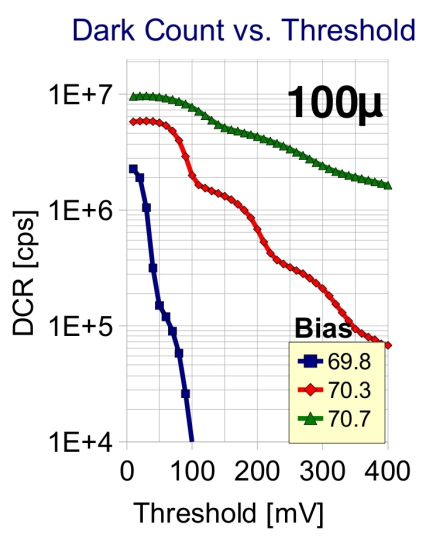

(c)

Figure 5: $100 \mu \mathrm{m}$ SPAD size: (a) bias voltage scan, (b) NINO threshold voltage scan, (c) DCR scan

Comparing the 3 different types $(25 \mu \mathrm{m}, 50 \mu \mathrm{m}$ and $100 \mu \mathrm{m})$ in Tab. 2 and Fig.6,a the $50 \mu$ gives best results with CTR values of 142ps FWHM at the minimum. Despite its good fill factor of $78.5 \%$ the $100 \mu \mathrm{m}$ type is only slightly better than the $25 \mu \mathrm{m}$ type. Obviously the $100 \mu \mathrm{m}$ type cannot be operated at optimum bias voltage because of its rapid increase in DCR, Fig.6,b. The $25 \mu \mathrm{m}$ type shows a CTR of 202ps FWHM and is thus about a factor $\sqrt{2}$ worse than the $50 \mu \mathrm{m}$ type. This factor could be explained by photon-statistics, because of its fill factor being $30.8 \%$ and thus half of the $50 \mu \mathrm{m}$ type.

\begin{tabular}{cccc||cccc}
\hline $\begin{array}{c}\text { Type: } \\
\text { S10931 }\end{array}$ & $\begin{array}{c}\text { SPAD } \\
\text { size } \\
\left(\mu \mathrm{m}^{2}\right)\end{array}$ & $\begin{array}{c}\text { Fill } \\
\text { Factor } \\
(\%)\end{array}$ & $\begin{array}{c}\text { Break down } \\
\text { voltage } \\
(\mathrm{V})\end{array}$ & $\begin{array}{c}\text { NINO } \\
\text { threshold } \\
(\mathrm{mV})\end{array}$ & $\begin{array}{c}\text { Bias } \\
\text { Voltage } \\
(\mathrm{V})\end{array}$ & $\begin{array}{c}\text { DCR at } \\
\text { bias voltage } \\
(\mathrm{Mcps})\end{array}$ & $\begin{array}{c}\text { Best CTR } \\
\text { FWHM } \\
(\mathrm{ps})\end{array}$ \\
\hline \hline$-025 \mathrm{P}$ & $25 \times 25$ & 30.8 & 69.2 & 40 & 73.0 & 4.55 & $202 \pm 4$ \\
$-050 \mathrm{P}$ & $50 \times 50$ & 61.5 & 70.5 & 40 & 72.2 & 0.88 & $142 \pm 4$ \\
$-100 \mathrm{P}$ & $100 \times 100$ & 78.5 & 69.3 & 40 & 70.3 & 5.85 & $192 \pm 8$ \\
\hline
\end{tabular}

Table 2: Minimal CTR achieved for different HAMAMATSU MPPCs with $3 \times 3 \mathrm{~mm}^{2}$ active area coupled to $2 \times 2 \times 5 \mathrm{~mm}^{3} \mathrm{LSO}: \mathrm{Ce}: \mathrm{Ca}$. Temperature at $20^{\circ} \mathrm{C}$ for all measurements. 


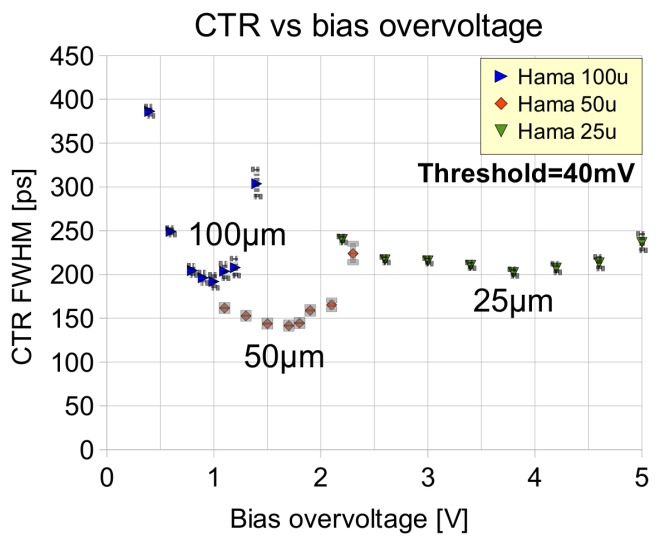

(a)

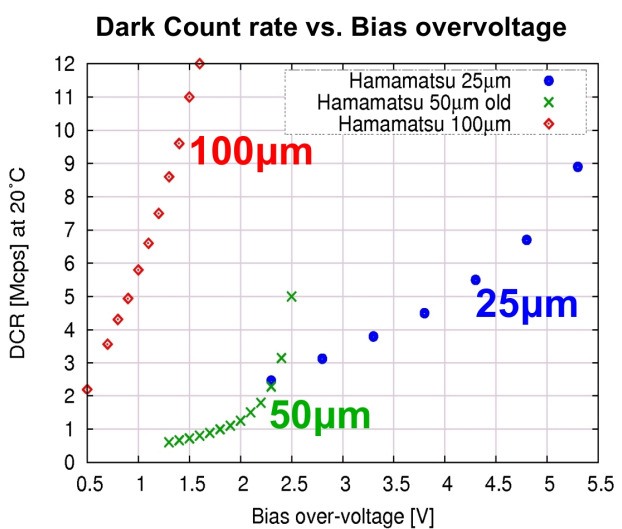

(b)

Figure 6: Summary of all 3 investigated SiPMs (a) bias voltage scan at same NINO threshold of $40 \mathrm{mV}$ and (b) DCR versus bias over-voltage

\section{Differences between production batches}

Variation of the timing behaviour between different production batches is an important consideration. We investigated 3 different production batches of the $50 \mu \mathrm{m}$ type received in June 2010 , May 2011 and January 2012. One special selected device with a lower DCR allowed to investigate the influence of DCR on the CTR at fixed temperature.

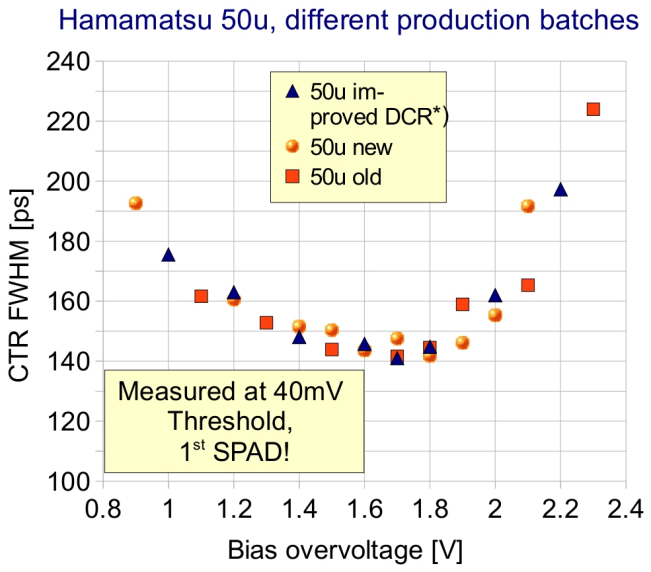

(a)

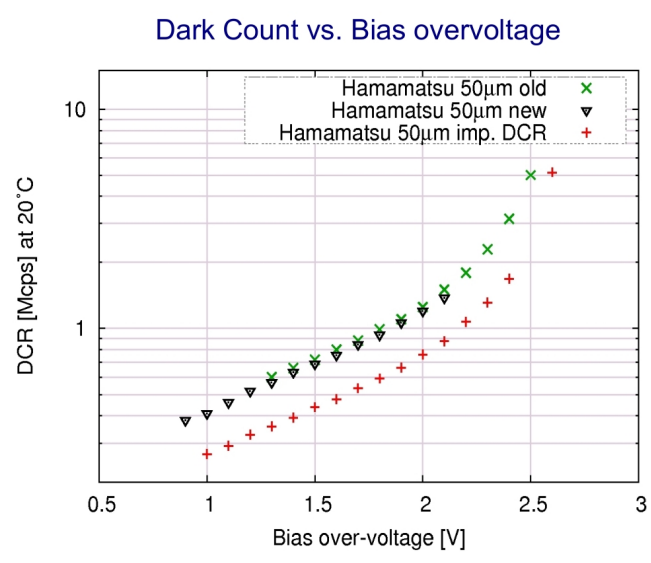

(b)

Figure 7: Measurements of 3 different batches: (a) CTR vs. bias overvoltage and (b) according DCR vs. bias overvoltage

We observed that the different production batches yielded the same timing behaviour when coupled to a LSO:Ce:Ca scintillation crystal with 5mm length, Fig.7. In our setup we did not see any improvement of time resolution despite reducing the DCR by about a factor 2 . This leads to the assumption that for the $50 \mu \mathrm{m}$ type other factors have a higher influence on time resolution. However it should be noticed that for the $50 \mu \mathrm{m}$ type the DCR is already very low, even at high overvoltages, suggesting a more refined production process compared to the $25 \mu \mathrm{m}$ and $100 \mu \mathrm{m}$ type. 


\section{Summary and Conclusion}

We investigated the timing resolution achievable with different types of SiPMs with different SPAD sizes and production batches. The $50 \mu \mathrm{m}$ SPAD size type gives best results among the different SPAD size types tested. Different production batches lead to same results in CTR, and dark count seems to play an inferior role if below a certain threshold.

\section{Acknowledgments}

We wish to thank Yuri Musienko for lending us the specially selected low dark count $50 \mu \mathrm{m}$ Hamamatsu SiPMs. In addition we want to thank Alain Machard for building the dark boxes and Dominique Deyrail for preparing the crystals.

\section{References}

[1] C.L. Kim,G.C. Wang and S. Dolinsky, "Multi-PiXel PHOtOn COUnTERS FOR TOF-PET DeteCtORS AND ITS CHALLENGES", IEEE Trans. Nucl. Sci., vol. 56, no. 5, pp. 2580-2585, Oct. 2009

[2] R. Vinke, H. Lohner, D.R. Schaart, H.T. van Dam, S. Seifert, F.J. Beekman and P. Dendooven, “OptimizING THE TIMING RESOLUTION OF SIPM SENSORS FOR USE IN TOF-PET DETECTORS “, Nucl. Instrum, Methods Phys, Res., vol. A610, pp. 188-191,2009

[3] S. Gundacker, E. Auffray, B. Frisch, H. Hillemanns, P. Jarron, T. Meyer, K. Pauwels, P. Lecoq, “A SystemATIC Study to Optimize SiPM Photodetectors For Highest Time Resolution IN PET”, IEEE transaction on nuclear science, May 2012, Issue: 99, Early Access Articles

[4] T. Szczesniak, M. Moszynski, L. Swiderski, A. Nassalski, P. Lavoute and M. Kapusta, FAsT PHOTOMULTIPLIERS FOR TOF PET, IEEE Trans. Nucl. Sci., vol. 56, no. 1, pp. 173-181, Feb. 2009

[5] E.Auffray, B. Frisch, F. Graci, A. Ghezzi, S. Gundacker, H. Hillemanns, P. Jaron, T. Meyer, M. Paganoni, K. Pauwels, M. Pizzichemi, P. Lecoq, "A COMPREHENSIVE \& SYSTEMATIC STUDY OF COINCIDENCE TIME RESOLUTION AND LIGHT YIELD USING SCINTILLATORS OF DIFFERENT SIZE, WRAPPING AND DOPING“, NSS/MIC, 2011 IEEE, Valencia 23-29 Oct. 2011, pp. 64-71

[6] W.W. Moses and S.E. Derenzo, "Prospects For Time-of-Flight PET using LSO Scintillator", IEEE Trans. Nucl. Sci. NS-46, pp. 474-478, 1999

[7] S. Seifert, H.T van Dam, R. Vinke, P. Dendooven, H. Löhner, F.J. Beekman and D.R. Schaart, A Comprehensive Model to Predict the Timing Resolution of SiPM-Based Scintillation Detectors: Theory And EXPERIMENTAL VALidation, IEEE Trans. Nucl. Sci., vol. 59, no. 1, Feb. 2012

[8] F. Powolny, E. Auffray, H. Hillemanns, P. Jarron, P. Lecoq, T.C. Meyer and D. Moraes, “A NOvel TIME-BASED READOUT SCHEME FOR A COMBINED PET-CT DETECTOR USING APDs“, IEEE Trans. Nucl. Sci., vol 55, no. 5, pp. 2465-2474, Oct. 2008

[9] F. Anghinolfi, P. Jarron, F. Krummenacher, E. Usenko and M.C.S. Williams, "NINO: AN ULTRAFAST LOW-POWER FRONT-END AMPLIFIER DISCRIMINATOR FOR THE TIME-OF-FLIGHT DETECTOR IN THE ALICE EXPERIMENT“, IEEE Trans. Nucl. Sci., vol. 51, No. 5, pp. 1974-1978, Oct 2004

[10] P. Jarron, E. Auffray, S.E. Brunner, M. Despeisse, E. Garutti, M. Goettlich, H. Hillemanns, P. Lecoq, T. Meyer, F. Powolny, W. Shen, H.C. Schultz-Coulon and M.C.S. Williams, "TIME BASED READOUT OF A SILICON PHOTOMULTIPLIER (SIPM) FOR TIME OF FLIGHT POSITRON EMISSION TOMOGRAPHY (TOF-PET)“, Nuclear Science Symposium Conference record (NSS/MIC), 2009 IEEE, pp. 1212-1219 